\title{
(HYBRID) BARYONS: QUANTUM NUMBERS AND ADIABATIC POTENTIALS
}

\author{
Philip R. Page* \\ Theoretical Division, MS B283, Los Alamos National Laboratory, \\ Los Alamos, NM 87545, USA
}

\begin{abstract}
We construct (hybrid) baryons in the flux-tube model of Isgur and Paton. In the limit of adiabatic quark motion, we build proper eigenstates of orbital angular momentum and indicate the flavour, spin, chirality and $J^{P}$ of (hybrid) baryons. The adiabatic potential is calculated as a function of the quark positions.
\end{abstract}

\section{Introduction}

Hybrid baryons are bound states of three quarks with an explicit excitation in the gluon field of QCD. The construction of (hybrid) baryons in a model motivated from and consistent with lattice gayge theory, the non-relativistic flux-tube model of Isgur and Paton, was detailed in ref. 4 There we studied the detailed flux dynamics and built the flux hamiltonian. A minimal amount of quark motion is allowed in response to flux motion, in order to work in the centre of mass frame. Otherwise, we make the so-called "adiabatic" approximation, where the flux motion adjusts itself instantaneously to the motion of the quarks. The main result was that the lowest flux excitation can to a high degree of accuracy (about 5\%) be simulated by neglecting all flux-tube motions except the vibration of a junction. The junction acquires an effective mass from the motion of the remainder of the flux-tube and the quarks. The model is then simple: a junction is connected via a linear potential to the three quarks. The ground state of the junction motion corresponds to a conventional baryon and the various excited states to hybrid baryons.

\section{Quantum numbers of (hybrid) baryons}

The junction can move in three directions, and correspondingly be excited in three ways, giving the hybrid baryons $H_{1}, H_{2}$ and $H_{3}$. For each junction excitation, it is found that the junction wave function can be realized to be either totally symmetric, or totally antisymmetric under quark label exchange, indicated by $H^{S}$ and $H^{A}$ respectively.

The quantum numbers of the lowest-lying states that can be constructed on the adiabatic surfaces corresponding to each of the six hybrid baryons are indicated in Table 1 .

*prp@t5.lanl.gov. Work done in collaboration with Simon Capstick. 
Table 1. Quantum numbers of ground state (hybrid) baryons for the adiabatic surfaces $B, H_{1}, H_{2}$ and $H_{3}$. Here $B$ denotes the conventional baryon. The mass ordering is $B<$ $H_{1}<H_{2}<H_{3}$. In the absense of spin dependent forces all ground states corresponding to a given adiabatic surface are degenerate. The quantum number notation is $(N, \Delta)^{2 S+1} J^{P}$, where $N, \Delta$ is the flavour structure of the wave function (i.e. those of the conventional baryons $N, \Delta$ respectively) and $P$ the parity.

\begin{tabular}{|c||r|l|l|}
\hline (Hybrid) Baryon & Chirality & $L$ & $(N, \Delta)^{2 S+1} J^{P}$ \\
\hline$B$ & 1 & 0 & $N^{2} \frac{1}{2}^{+}, \Delta^{4} \frac{3}{2}^{+}$ \\
$H_{1}^{S}$ & 1 & 1 & $N^{2} \frac{1}{2}^{+}, N^{2} \frac{3}{2}^{+}, \Delta^{4} \frac{1}{2}^{+}, \Delta^{4} \frac{3}{2}^{+}, \Delta^{4} \frac{5}{2}^{+}$ \\
$H_{1}^{A}$ & 1 & 1 & $N^{2} \frac{1}{2}^{+}, N^{2} \frac{3}{2}^{+}$ \\
$H_{2}^{S}$ & 1 & 1 & $N^{2} \frac{1}{2}^{+}, N^{2} \frac{3}{2}^{+}, \Delta^{4} \frac{1}{2}^{+}, \Delta^{4} \frac{3}{2}^{+}, \Delta^{4} \frac{5}{2}^{+}$ \\
$H_{2}^{A}$ & 1 & 1 & $N^{2} \frac{1}{2}^{+}, N^{2} \frac{3}{2}^{+}$ \\
$H_{3}^{S}$ & -1 & 0 & $N^{2} \frac{1}{2}^{-}, \Delta^{4} \frac{3}{2}^{-}$ \\
$H_{3}^{A}$ & -1 & 0 & $N^{2} \frac{1}{2}^{-}$ \\
\hline
\end{tabular}

Since quarks are fermions, the wave function should be totally antisymmetric under quark label exchange, called the Pauli principle. The colour structure of hybrid baryons are taken to be identical to those of conventional baryons, i.e. it is totally antisymmetric under label exchange. This imposes constraints on the combination of flavour and non-relativistic spin $S$ of the three quarks that is allowed. The combinations are indicated in Table 1 .

"Chirality" gives the behaviour of the junction wave function under reflection in the plane spanned by the quarks, when the positions of the quarks are fixed.

Let $L$ be the orbital angular momentum of the quarks and the junction. It is possible to argue that $L=1$ for the ground state $H_{1}$ and $H_{2}$ hybrid baryons, while $L=0$ for the ground state conventional and $H_{3}$ hybrid baryons. The total angular momentum $\mathbf{J}=\mathbf{L}+\mathbf{S}$. Since $L=0$ for ground state conventional and $H_{3}$ hybrid baryons, $J=S$. Since $L=1$ for ground state $H_{1}, H_{2}$ hybrid baryons, $J=\frac{1}{2}, \frac{3}{2}$ for $S=\frac{1}{2}$, and $J=\frac{1}{2}, \frac{3}{2}, \frac{5}{2}$ for $S=\frac{3}{2}$. These assignments are indicated in Table 1 .

\section{The potential in which the quarks move}

We shall now calculate the junction energy ("adiabatic potential") for the ground and first excited states of the junction as a function of the quark positions.

Define

$$
\boldsymbol{\rho}=\frac{\mathbf{r}_{1}-\mathbf{r}_{2}}{\sqrt{2}} \quad \boldsymbol{\lambda}=\frac{\mathbf{r}_{1}+\mathbf{r}_{2}-2 \mathbf{r}_{3}}{\sqrt{6}} \quad \cos \theta_{\rho \lambda}=\frac{\boldsymbol{\rho} \cdot \boldsymbol{\lambda}}{\rho \lambda}
$$

where $\mathbf{r}_{i}$ denotes the positions of the quarks. The energy is a function of $\rho, \lambda$ and $\theta_{\rho \lambda}$.

The procedure for evaluating the conventional baryon potential is as follows. We numerically evaluate $V_{B}\left(l_{1}, l_{2}, l_{3}\right)$ by solving the Schrödinger Equation for the junction 


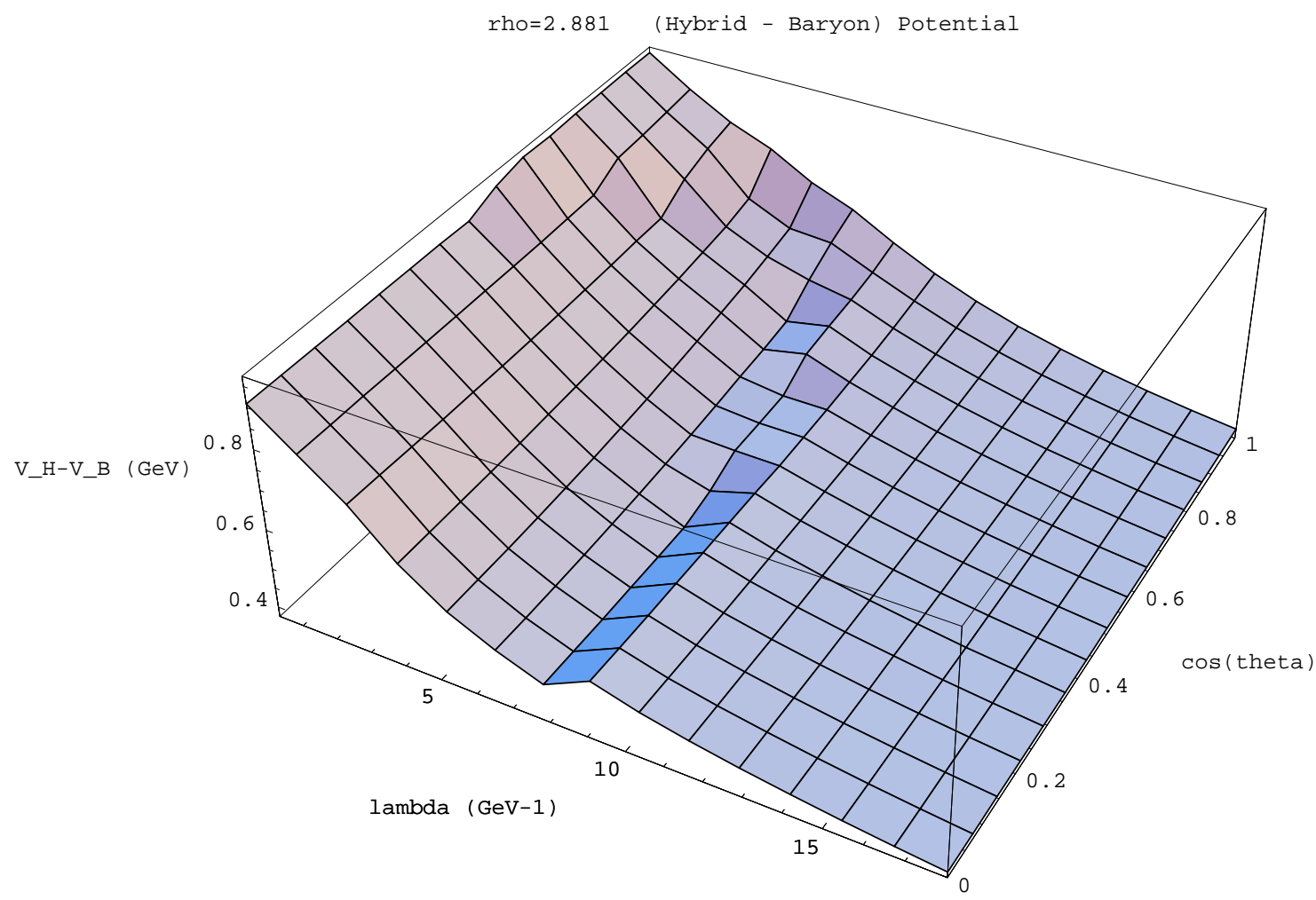

Fig. 1. Difference of the hybrid and conventional baryon potentials (in GeV) for $\rho=2.881$ $\mathrm{GeV}^{-1}$ as a function of $\lambda\left(\right.$ in $\left.\mathrm{GeV}^{-1}\right)$ and $\cos \theta_{\rho \lambda}$.

hamiltonian, $\left(\frac{1}{2} M_{\mathrm{eff}}^{\infty} \dot{\mathbf{r}}^{\mathbf{2}}+V_{\mathrm{J}}\right) \Psi_{B}(\mathbf{r})=V_{B}\left(l_{1}, l_{2}, l_{3}\right) \Psi_{B}(\mathbf{r})$, variationally using an ansatz ground state simple harmonic oscillator wave function. $M_{\text {eff }}^{\infty}$ is the effective mass of the junction in the limit where the flux-tubes between the junction and quarks are continuous strings. $V_{\mathrm{J}}$ is the linear potential between the junction and the three quarks. The hybrid baryon $H_{1}$ potential $V_{H_{1}}\left(l_{1}, l_{2}, l_{3}\right)$ is solved using $\left(\frac{1}{2} M_{\text {eff }^{\infty}}^{\infty} \dot{\mathbf{r}}^{2}+V_{\mathrm{J}}\right) \Psi_{H_{1}}(\mathbf{r})=$ $V_{H_{1}}\left(l_{1}, l_{2}, l_{3}\right) \Psi_{H_{1}}(\mathbf{r})$ with a first excited state simple harmonic oscillator wave function as an ansatz.

The difference between the hybrid and conventional baryon potentials is plotted in Figures 1 - 2. Since the potentials are functions of $\rho, \lambda$ and $\theta_{\rho \lambda}$, one of the variables is held fixed at a typical value for clarity of presentation.

The (hybrid) baryon potential can be seen to increase when $\rho \lambda$ is small. Numerically, the ratio of the hybrid to baryon potential is found to be $1.44-1.6$ for all $\rho, \lambda$ and $\theta_{\rho \lambda}$.

A preliminary estimate of the ground state $H_{1}$ hybrid baryon mass of $\sim 2 \mathrm{GeV}$ has also been made by adding the difference between the hybrid and conventional baryon potentials to the phenomenologically successful baryon potential used in ref.2 


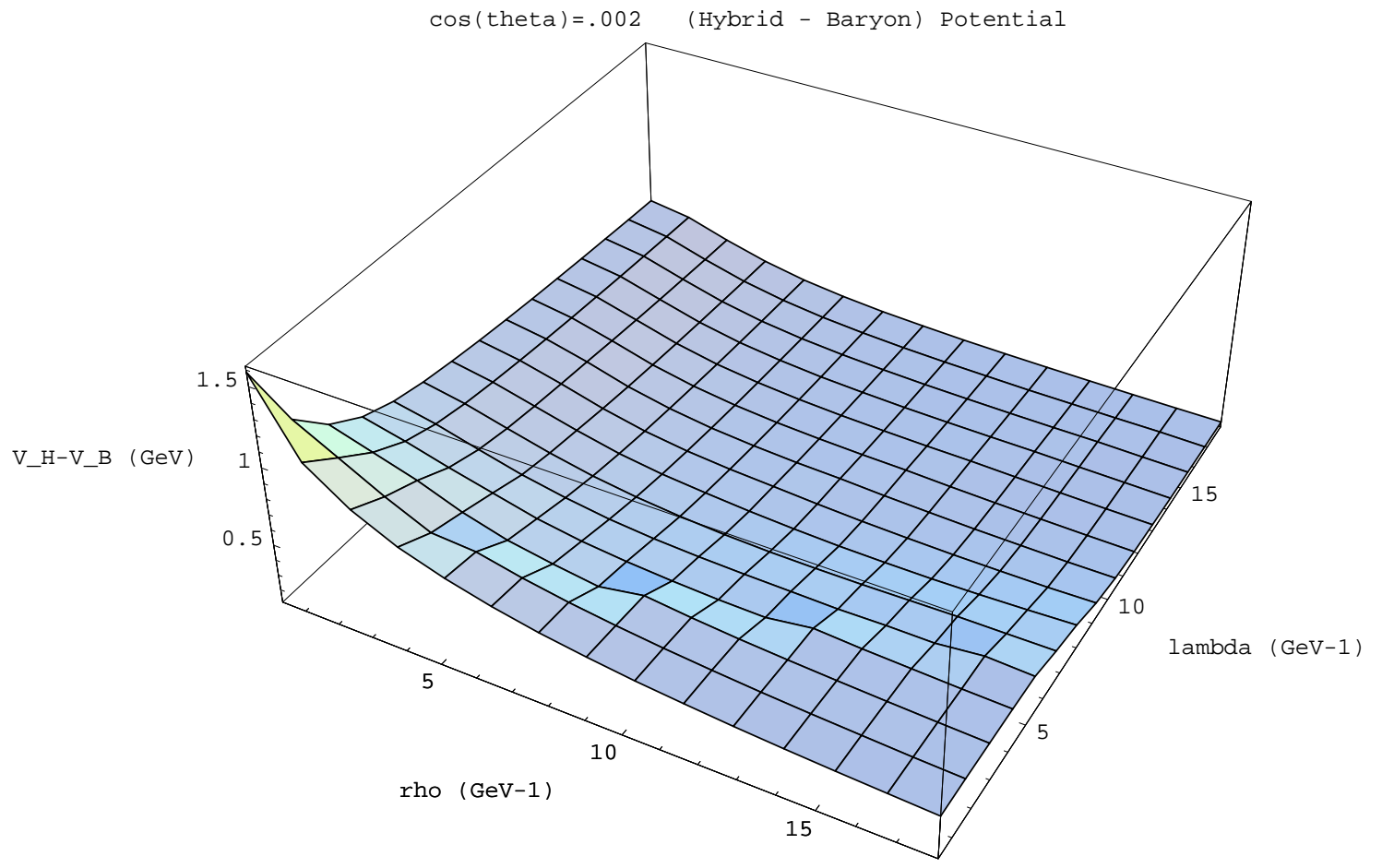

Fig. 2. Difference of the hybrid and conventional baryon potentials for $\cos \theta_{\rho \lambda}=0.002$ as a function of $\rho$ and $\lambda$ (in $\mathrm{GeV}^{-1}$ ).

\section{Conclusions}

The spin and flavour structure of the six hybrid baryons have been specified. Exchange symmetry constrains the spin and flavour of the (hybrid) baryon wave function. The orbital angular momentum of the low-lying hybrid baryon is argued to be unity. The adiabatic potentials have been calculated numerically. The low-lying hybrid baryon mass has been estimated numerically.

\section{References}

1. P.R. Page, Proc. of "Seventh International Conference on Hadron Spectroscopy" (HADRON '97), 25-30 August 1997, Upton, N.Y., U.S.A., eds. S.-U. Chung, H.J. Willutzki, p. 553, American Institute of Physics.

2. S. Capstick, N. Isgur, Phys. Rev. D34 (1986) 2809. 